\title{
AOIR
}

Selected Papers of \#AolR2020:

The 22nd Annual Conference of the

Association of Internet Researchers Virtual Event / 13-16 Oct 2021

\section{TWEETING BACK: EXPLORING BLACK FEMINIST HASHTAG COMMUNITIES ON TWITTER \& INSTAGRAM}

\author{
Chelsea Peterson-Salahuddin \\ Northwestern University
}

\section{Motivation}

Hashtag discussions have become a key site of intellectual production for Black feminist theorizing; however, the nuances of these hashtag networks remain underexplored (Conley, 2017; Jones, 2019). Black feminist theory is a line of intellectual thought developed from Black women's unique lived experiences at the varying intersections of race, gender, sexuality, and class, privileging the link between lived experience and theorizing (Collins, 2000). While scholars have previously explored the use of hashtags by Black women to talk about their lived experiences within the larger context of Black Twitter (Clark, 2014), and theorized Black feminist hashtags through content analysis of social media posts (Conley, 2017; Jackson, Bailey, \& Welles, 2020), these studies have not accounted for the experiences and motivations of social media users who engage specifically in Black feminist conversations. This study aimed to deepen this previous research by speaking with those who participate in these networked conversations to understand the distinct nuances of their engagement experiences.

I term these conversational communities Platformed Black Feminist Communities; social media-based communities often formed around hashtags that discuss Black women's unique lived experiences. Drawing from definitions of Black Twitter (Brock, 2020), this study's understanding of Platformed Black Feminist Communities is not synonymous with all Black women on social media but rather a specific group of users who actively use social media to engage in discussions about common cultural understandings of Black womanhood. Finally, my use of the word "platformed" draws from scholarship on platformization as an organizing economic and infrastructural model online to denote how these community interactions are shaped by platform logics and imperatives (Helmond, 2015; Nieborg \& Powell, 2018).

In this study, I had three main research questions:

1. How do individuals who participate in black feminist hashtag conversations on Twitter and Instagram understand their networked interactions on these platforms?

2. Why do individuals choose to take part in Black feminist hashtag conversations on Twitter/Instagram? 
3. What is at stake in how black feminist hashtag conversations circulate on Twitter and Instagram?

\section{Methods}

To answer these research questions, I conducted in-depth, semi-structured interviews with individuals that created, popularized, or participated in Black feminist hashtag conversations on Twitter or Instagram, two social media platforms on which hashtags, and Black feminist hashtags, in particular, have been prevalent (Jones, 2019; Pham, 2015).

Since a key way Platfromed Black Feminist Communities are discoverable is through black feminist hashtags, to begin recruitment, I searched Google and Google Scholar using the terms 'black,' 'feminist,' and 'hashtag,' to identified hashtags on Twitter or Instagram that previously had been identified as 'black feminist' in focus, or could arguably be called 'black feminist.' I also solicited suggestions of black feminist hashtags from other intersectional media scholars. I then removed all hashtags from the data set whose initial creators I could not verifiably identify.

I then directly contacted all the individual(s) who created or popularized a hashtag on my list to inquire if they would participate in the study. After these initial interviews, I employed a snowball sampling method informed by network analysis, asking the hashtag creator to refer me to three to five other people from their own Twitter or Instagram network who took part in their hashtag conversation. I employed this snowball sampling method with every subsequent interviewee. By employing this strategy, I aimed to elucidate how hashtag meaning and usage could shift through dispersion in a network over time while maintaining a qualitative interview methodology (Korn, 2015).

This sampling strategy resulted in interviews with 21 individuals who engaged in at least one of eight Black feminist hashtag discussions on Twitter or Instagram. All participants self-identified as Black women, despite the open-ended snowball sampling prompt, potentially indicating that the majority of individuals who take part in these Black feminist hashtag conversations on social media are, in fact, Black women.

\section{Findings}

\section{R1: How do individuals who participate in black feminist hashtag conversations on Twitter and Instagram understand their networked interactions on these platforms?}

Almost all of my participants joined these platforms when they were first launched. While some participants initially joined these platforms because of pre-existing social connections, many joined to find new communities of Black women. On social media, participants would often connect with other Black women and femmes through collective conversation around popular culture issues related to the intersection of race and 
gender. Through these discussions, participants formed online communities based on ethics of care and collective acts of 'talking back' to mainstream depictions of Black womanhood (Collins, 1989; hooks, 1989).

However, more recently, the dynamics of these networks have shifted. Socio-political shifts included the 2016 election and the rise of the Black Lives Matter Movement, led to more instances of participants facing misogynoir-based harassment (Bailey \& Trudy, 2018). As a result, participants noted their behavior on these platforms is much more tempered - many note they often say less and block more.

\section{R2: Why do individuals choose to take part in Black feminist hashtag conversations on Twitter \& Instagram?}

All eight hashtag conversations explored in this study were created in response to situations that highlighted the particular nuances of participants ' lived experiences as Black women, especially when they contradicted dominant narratives about Black womanhood. Collectively, These discussions fostered collective acts of what Black feminist scholar bell hooks (1989) terms "talking back," speech act of agency, and selfdefinition as a form of resistance.

The main reasons participants chose to engage in these conversations were: the way the discussions centered on the experiences of Black women, the hashtag message resonated with their own experiences, and a belief in the mission or the purpose of the hashtag. In all of these instances, by participating in these hashtags, participants sought to publicly affirm and bolster these conversations by acknowledging and amplifying them as fellow Black women, highlight the highly supportive nature of Platformed Black Feminist Hashtag Communities.

\section{R3: What is at stake in how black feminist hashtag conversations circulate on Twitter and Instagram?}

Due to the unforeseen ways hashtags can spread online, many of the hashtag creators and popularizers noted how their hashtags meaning or use had changed or expanded over time. Also, with virality came the threat of erasure - several participants who had created or popularized hashtags noted their erasure from the hashtag's origin.

\section{Discussion}

Platformed Black Feminist Communities offer a critical space through which Black women and femmes can discuss culturally specific topics through the shared yet intersectional lens. Yet, they can also open Black women users up to new forms of harassment. Further, the nature of virality on social media can often lead to these hashtag discussions being misused, appropriated, and plagiarized, replicating historical instances where Black women's women's work has been overlooked, copied, and stolen (Cooper, 2017). These findings point to how social media both opens up new liberatory spaces for Black feminist expression and can expose the Black women who participate in these conversations to new forms of harm and harassment. In this way, the dynamics of Platformed Black Feminist Community members engagement 
illuminated through this study highlight the interdependent and complex relationship between social media and Black feminist theorizing - with social media comes both new spaces of intellectual production and new potential spaces of harm.

\section{Work Cited}

Brock, A. (2020). Distributed blackness. New York, NY: NYU Press.

Clark, M. (2014). To Tweet Our Own Cause: a mixed-methods study of the online phenomenon "Black Twitter." (Unpublished doctoral thesis). The University of North Carolina at Chapel Hill, Chapel Hill, North Carolina.

Collins, P.H. (2000). Black Feminist Thought (2 ed.) New York: Routledge.

Conley, T.L. (2017). Decoding Black Feminist Hashtags as Becoming. The Black Scholar, 47(3), 22-32.

Cooper, B. (2017). Beyond Respectability: The Intellectual Thought of Race Women. Champaign, IL: University of Illinois Press.

Helmond, A. (2015). The Platformization of the Web: Making Web Data Platform Ready. Social Media + Society.

hooks, b. (1989). Talking Back: Thinking feminist, thinking Black. Boston, MA: South End Press.

Jackson, S., Bailey, M., \& Welles, B.F. (2020). Hashtag activism. Cambridge: MIT University Press.

Jones, F. (2019). Reclaiming Our Space. Boston, MA: Beacon Press.

Korn, J. (2015). \#FuckProp8: How Temporary Virtual Communities around Politics and Sexuality Pop Up, Come Out, Provide Support, and Taper Off. In N. Rumbukkana (Ed), Hashtag publics and the power of political discursive networks (pp. 204-217). New York, NY: Peter Lang.

Nieborg, D. B., \& Poell, T. (2018). The platformization of cultural production: Theorizing the contingent cultural commodity. New Media \& Society, 20(11), 4275-4292.

Pham, M.T. (2015). "I Click and Post and Breathe, Waiting for Others to See What I See": On \#FeministSelfies, Outfit Photos, and Networked Vanity. Fashion Theory, 19(2), pp. 221-242. 